\title{
ON EULER'S CRITERION
}

EMMA LEHMER

(rec. 2 April 1959)

Euler's criterion states that if $p$ is a prime then

$$
D^{f} \equiv \mathbf{1}(\bmod p), p=k f+1,
$$

if and only if $D$ is a $k$-th power residue of $p$.

However if (1) does not hold then

$$
D^{f} \equiv \alpha_{k}(\bmod p), \alpha_{k} \neq \equiv 1(\bmod p),
$$

where $\alpha_{k}$ is some $k$-th root of unity modulo $p$.

For $k=2$ it is obvious that $\alpha_{k}=-1$ and we have the usual congruence for the Legendre symbol, namely

$$
D^{(p-1) / 2} \equiv\left(\frac{D}{p}\right) \quad(\bmod p)
$$

For $k>2$ there seems to have been no attempt in the literature to specify which $\alpha_{k}$ corresponds to a given $D$. This is probably due to the fact that in general one would not expect to be able to distinguish between primitive $k$-th roots of unity. The possibility of this determination for $k=3$ and $D=2$ was suggested by empirical results of N.Y. Wilson which can be reduced to our criterion (24). Explicit results will be given for $D=2$ also with $k=3,4,5$, and 8 as well as some general congruence relations involving the so called Jacobsthal sums

$$
\phi_{k}(D)=\sum_{\nu=1}^{p-1}\left(\frac{\nu}{p}\right)\left(\frac{\nu^{k}+D}{p}\right) .
$$

We consider the sum (4) as a congruence modulo $p$. Using (3) we obtain

$$
\phi_{k}(D) \equiv \sum_{\nu=1}^{p-1} \nu^{(p-1) / 2}\left(\nu^{k}+D\right)^{(p-1) / 2} \equiv \sum_{\mu=0}^{(p-1) / 2}\left(\begin{array}{c}
(p-1) / 2 \\
\mu
\end{array}\right) D^{\mu} S_{k}(\mu)
$$

where

$$
S_{k}(\mu)=\sum_{\nu=1}^{p-1} \nu^{\frac{1}{2}(k+1)(p-1)-k \mu} \equiv \begin{cases}\sum_{\nu=1}^{p-1} \nu^{-k \mu} & \text { if } k \text { is odd } \\ \sum_{\nu=1}^{p-1} \nu^{(p-1) / 2-k \mu} & \text { if } k \text { is even }\end{cases}
$$


Hence if $k$ is odd

$$
S_{k}(\mu) \equiv\left\{\begin{array}{cc}
-1 & (\bmod p) \text { if } \mu=m f \\
0 & (\bmod p) \text { otherwise, }
\end{array}\right.
$$

while if $k$ is even

$$
S_{k}(\mu) \equiv\left\{\begin{array}{cc}
-1 & (\bmod p) \text { if } \mu=(2 m+1) f / 2 \\
0 & (\bmod p) \text { otherwise. }
\end{array}\right.
$$

Combining these results we have

(7) $\phi_{k}(D) \equiv\left\{\begin{array}{l}-\sum_{m=0}^{(k-1) / 2} D^{m f}\left(\begin{array}{c}(p-1) / 2 \\ m f\end{array}\right)(\bmod p) \text { for } k \text { odd } \\ -\sum_{m=0}^{(k-2) / 2} D^{(2 m+1) f / 2}\left(\begin{array}{c}(p-1) / 2 \\ (2 m+1) f / 2\end{array}\right)(\bmod p), k \text { and } f \text { even } \\ 0(\bmod p) \text { for } k \text { even, } f \text { odd. }\end{array}\right.$

This congruence can be found in a slightly different form in Whiteman [1].

For $k=2, f / 2=(p-1) / 4, p=4 n+1=a^{2}+b^{2}, a \equiv 1(\bmod 4)$ congruence (7) becomes

$$
\phi_{2}(D) \equiv-D^{(p-1) / 4}\left(\begin{array}{l}
(p-1) / 2 \\
(p-1) / 4
\end{array}\right) \quad(\bmod p) .
$$

Putting $D=1$ we get the well known result of Gauss

$$
\left(\begin{array}{l}
(p-1) / 2 \\
(p-1) / 4
\end{array}\right) \equiv-\phi_{2}(1)=2 a(\bmod p) .
$$

Therefore

$$
D^{(p-1) / 4} \equiv \phi_{2}(D) / \phi_{2}(1)(\bmod p) .
$$

Jacobsthal [2] proved in his dissertation that if $D \equiv m^{2}(\bmod p)$

$$
\phi_{2}(D)=\phi_{2}\left(m^{2}\right)=\left(\frac{m}{p}\right) \phi_{2}(1)=-\left(\frac{m}{p}\right) 2 a .
$$

Substituting this into (10) gives (3). In case $D$ is not a quadratic residue Jacobsthal was only able to prove that

$$
\phi_{2}(D)= \pm 2 b \text { if }\left(\frac{D}{p}\right)=-1,
$$

which is insufficient for our purposes. In another paper [3] we were able to improve on this result if 2 is not a quartic residue of $p=4 n+1$ as follows: 
(13) $\phi_{2}(D)=-2 b\left(\frac{m}{p}\right)$ where $\left\{\begin{array}{c}D \equiv 2 m^{2}(\bmod p),\left(\frac{2}{p}\right)=-1, \\ b / 2 \equiv 1(\bmod 4) \\ D \equiv \sqrt{ } 2 m^{2}(\bmod p),\left(\frac{2}{p}\right)=1, \\ b / 4 \equiv(-1)^{n}(\bmod 4) \text {. }\end{array}\right.$

Substituting these values into (10) we obtain in case 2 is not a quartic residue

$$
D^{(p-1) / 4} \equiv\left(\frac{m}{p}\right) b / a(\bmod p)
$$

in case either $D \equiv 2 m^{2}$ and $\left(\frac{2}{p}\right)=-1$ with $b / 2 \equiv 1(\bmod 4)$

$$
\text { or } D=\sqrt{ } 2 m^{2} \text { and }\left(\frac{2}{p}\right)=+1 \text { with } b / 4 \equiv(-1)^{n}(\bmod 4) \text {. }
$$

In the case $D=2$, the results are much more explicit. It is well known that 2 is a quartic residue of $p$ if and only if $b \equiv 0(\bmod 8)$. If 2 is a quadratic but not quartic residue then $b \equiv 4(\bmod 8)$, in the remaining cases $b$ is oddly even and we can take as above $b / 2 \equiv 1(\bmod 4)$ and state our criterion as follows:

If $p=4 n+1=a^{2}+b^{2}, a \equiv 1(\bmod 4)$, then

$$
2^{(p-1) / 4} \equiv\left\{\begin{array}{l}
(-1)^{b / 4}(\bmod p) \text { if } b \equiv 0 \quad(\bmod 4) \\
b / a(\bmod p) \text { otherwise }[b / 2 \equiv 1 \quad(\bmod 4)]
\end{array}\right.
$$

Next let $k=3, f=(p-1) / 3$. In this case the sum (7). reduces to

$$
\phi_{3}(D) \equiv-1-D^{f}\left(\begin{array}{c}
(p-1) / 2 \\
f
\end{array}\right) \quad(\bmod p) \text {. }
$$

Letting $D=1$ we have

$$
\left(\begin{array}{l}
(p-1) / 2 \\
(p-1) / 3
\end{array}\right) \equiv-1-\phi_{3}(1) \quad(\bmod p) .
$$

Substituting this back into (16) we obtain

$$
D^{(p-1) / 3} \equiv\left(\phi_{3}(D)+1\right) /\left(\phi_{3}(1)+1\right) \quad(\bmod p)
$$

Since $\phi_{3}(D)=\phi_{3}(1)$ if $D$ is a cubic residue the above congruence reduces in this case to Euler's criterion. If $D$ is not a cubic residue however the general formula for $\phi_{3}(D)$ contains an ambiguity of sign. We were able to determine this sign in a previous paper [3] under the condition that 2 is not a cubic residue. Let

$$
p=A^{2}+3 B^{2} \text { and } 4 p=L^{2}+27 M^{2}, A \equiv L \equiv 1 \quad(\bmod 3)
$$


then

$$
2^{(p-1) / 3} \equiv 1 / 4^{(p-1) / 3} \equiv-2 A / L \quad(\bmod p)
$$

since $[4]$

$$
\phi_{3}(D)=\phi_{3}(1)=-(2 A+1) \text { if } D=m^{3}
$$

while

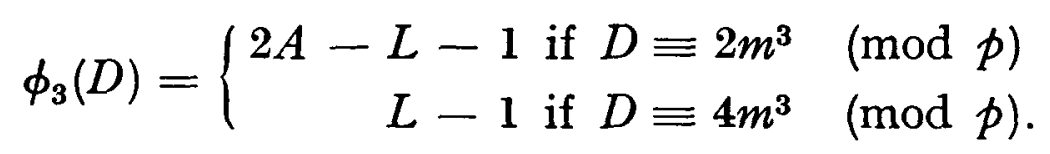

It might be worth recalling that 2 is a cubic residue of $p$ if and only if $^{*} L \equiv 0(\bmod 2)$; but this implies $B \equiv 0(\bmod 3)$ and $L=-2 A$, $B= \pm 3 M$; hence (19) reduces to (1). If 2 is not a cubic residue then $B \not \equiv 0(\bmod 3)$ and we may choose $B \equiv 1(\bmod 3)$. Then it can be easily verified that the two forms are related by $L=A+3 B, A-B= \pm 3 M$. Hence we can eliminate $L$ in (21), thus obtaining our result in terms of a single form as follows:

If $p=A^{2}+3 B^{2}, A \equiv B \equiv 1(\bmod 3)$ and 2 is not a cubic residue, then,

$$
\varphi_{3}(D)= \begin{cases}A-3 B-1 \text { if } D=2 m^{3} & (\bmod p) \\ A+3 B-1 \text { if } D=4 m^{3} & (\bmod p)\end{cases}
$$

Substituting this and (20) into (18) we obtain

$$
D^{(p-1) / 3} \equiv\left\{\begin{array}{cll}
1 & \text { if } D \equiv m^{3} & (\bmod p) \\
(-A+3 B) / 2 A & \text { if } D \equiv 2 m^{3} & (\bmod p) \\
-(A+3 B) / 2 A & \text { if } D \equiv 4 m^{3} & (\bmod p)
\end{array}\right.
$$

It might be worth noting that $(-A \pm 3 B) / 2 A \equiv(\mp A-B) / 2 B(\bmod p)$. This can be verified by cross multiplication using $A^{2} \equiv-3 B^{2}(\bmod p)$. For $D=2$ we get the following explicit result:

$$
2^{(p-1) / 3} \equiv\left\{\begin{array}{rlrl}
1 \quad(\bmod p) & \text { if } B & \equiv 0 & (\bmod 3) \\
(3 B-A) / 2 A \equiv-(A+B) / 2 B[B & \equiv 1 & (\bmod 3)]
\end{array}\right.
$$

By (20) and (17) we get the well known result.

$$
\left(\begin{array}{l}
(p-1) / 2 \\
(p-1) / 3
\end{array}\right) \equiv 2 A(\bmod p), \quad A \equiv 1(\bmod 3) .
$$

For $k=5, f=(p-1) / 5$, congruence (7) gives

$$
-\left[1+\phi_{5}(D)\right]=D^{f}\left(\begin{array}{c}
(p-1) / 2 \\
f
\end{array}\right)+D^{2 f}\left(\begin{array}{c}
(p-1) / 2 \\
2 f
\end{array}\right) \quad(\bmod p) .
$$

We write this congruence for $D=4 d^{\nu}, \nu=0,1,2,3,4$, where $d$ is any 
quintic non-residue of $p$ and let

$$
\begin{aligned}
c_{\nu} & =-\left[1+\phi_{5}\left(4 d^{\nu}\right)\right], \quad(v=0,1,2,3,4), \\
\gamma_{1} & =4^{f}\left(\begin{array}{c}
(p-1) / 2 \\
f
\end{array}\right) \equiv\left(\begin{array}{c}
2 f \\
f
\end{array}\right) \quad(\bmod p),
\end{aligned}
$$

and

$$
\gamma_{2}=4^{2 f}\left(\begin{array}{c}
(p-1) / 2 \\
2 f
\end{array}\right) \equiv\left(\begin{array}{c}
4 f \\
2 f
\end{array}\right) \equiv\left(\begin{array}{c}
3 f \\
f
\end{array}\right) \quad(\bmod p) .
$$

Then (26) can be replaced by the system of congruences

$$
c_{\nu} \equiv \gamma_{1} d^{\nu f}+\gamma_{2} d^{2 v f} \quad(\bmod p) \quad(v=0,1,2,3,4) .
$$

This system can be solved for $\gamma_{1}$ and $\gamma_{2}$ as follows. We note that

$$
\begin{gathered}
c_{1}-c_{2}-c_{3}+c_{4} \equiv\left(d^{f}-d^{2 f}-d^{3 f}+d^{4 f}\right)\left(\gamma_{1}-\gamma_{2}\right) \quad(\bmod p), \\
c_{1} c_{4}+c_{2} c_{3} \equiv 2\left(\gamma_{1}^{2}+\gamma_{2}^{2}\right)-\gamma_{2} \gamma_{2} \equiv 2 c_{0}^{2}-5 \gamma_{1} \gamma_{2} \quad(\bmod p),
\end{gathered}
$$

since $\gamma_{1}+\gamma_{2} \equiv c_{0}(\bmod p)$,

$$
\begin{aligned}
c_{1} c_{4}-c_{2} c_{3} & \equiv\left(d^{f}-d^{2 f}-d^{3 f}+d^{4 f}\right) \gamma_{1} \gamma_{2} \\
& \equiv\left(d^{f}-d^{2 f}-d^{3 f}+d^{4 f}\right)\left(2 c_{0}^{2}-c_{1} c_{4}-c_{2} c_{3}\right) / 5 \quad(\bmod p),
\end{aligned}
$$

by (32). Hence by (31) and (33)

(34) $\gamma_{1}-\gamma_{2} \equiv\left(c_{1}-c_{2}-c_{3}+c_{4}\right)\left(2 c_{0}^{2}-c_{1} c_{4}-c_{2} c_{3}\right) / 5\left(c_{1} c_{4}-c_{2} c_{3}\right)(\bmod p)$.

Therefore,

$$
\gamma_{1} \equiv\left(\begin{array}{c}
2 f \\
f
\end{array}\right) \equiv \frac{1}{2}\left[c_{0}+\left(c_{1}-c_{2}-c_{3}+c_{4}\right)\left(2 c_{0}^{2}-c_{1} c_{4}-c_{2} c_{3}\right) / 5\left(c_{1} c_{4}-c_{2} c_{3}\right)\right]
$$

and

(36) $\gamma_{2} \equiv\left(\begin{array}{c}3 f \\ f\end{array}\right) \equiv \frac{1}{2}\left[c_{0}-\left(c_{1}-c_{2}-c_{3}+c_{4}\right)\left(2 c_{0}^{2}-c_{1} c_{4}-c_{2} c_{3}\right) / 5\left(c_{1} c_{4}-c_{2} c_{3}\right)\right]$ $(\bmod p)$.

We now recall that $\phi_{5}\left(4 d^{\nu}\right)$ and therefore the $c_{\nu}$ 's can be evaluated [1] in terms of the quadratic partition

$$
\left\{\begin{array}{l}
16 p=x^{2}+50 u^{2}+50 v^{2}+125 w^{2} \\
x w=v^{2}-u^{2}-4 u v,
\end{array} \quad x \equiv 1(\bmod 5),\right.
$$

as follows:

$$
\begin{aligned}
c_{0} & =-\left[1+\phi_{5}(4)\right]=-x \\
4 c_{1} & =x-25 w-10(u+2 v) \\
4 c_{2} & =x+25 w-10(2 u-v) \\
4 c_{3} & =x+25 w+10(2 u-v) \\
4 c_{4} & =x-25 w+10(u+2 v) .
\end{aligned}
$$


Therefore $c_{1}-c_{2}-c_{3}+c_{4}=-25 w$, while

$$
4\left(c_{1} c_{4}+c_{2} c_{3}\right)=3 x^{2}+625 w^{2}
$$

and

$$
c_{1} c_{4}-c_{2} c_{3}=25\left(u^{2}-v^{2}-u v\right) \equiv-25(x w+5 u v) \quad(\bmod p) .
$$

Hence (35) and (36) become

$$
\left(\begin{array}{c}
2 f \\
f
\end{array}\right) \equiv \frac{1}{2}\left[-x+\frac{w\left(x^{2}-125 w^{2}\right)}{4(x w+5 u v)}\right] \quad(\bmod p)
$$

and

$$
\left(\begin{array}{c}
3 f \\
f
\end{array}\right) \equiv \frac{1}{2}\left[-x-\frac{w\left(x^{2}-125 w^{2}\right)}{4(x w+5 u v)}\right] \quad(\bmod p) .
$$

We note that these results are unambiguous since the same answer is obtained by substituting either of the four solutions of (37), namely

$$
(x, u, v, w) ; \quad(x,-u,-v, w) ; \quad(x, v,-u,-w) ; \quad(x,-v, u,-w) .
$$

Knowing $\gamma_{2}$ we can solve the system (30) for $d^{v f}$ as follows. Writing $2 v$ and $3 v$ for $v$ in (30) we obtain

$$
\begin{aligned}
d^{\nu f} c_{2 \nu} & \equiv \gamma_{1} d^{3 \nu f}+\gamma_{2} & & (\bmod p) \\
c_{3 \nu} & \equiv \gamma_{1} d^{3 \nu f}+\gamma_{2} d^{\nu f} & & (\bmod p) .
\end{aligned}
$$

Hence subtracting,

$$
d^{\nu f} \equiv\left(c_{3 \nu}+\gamma_{2}\right) /\left(c_{2 \nu}+\gamma_{2}\right) \quad(\bmod p) .
$$

The last expression is not devoid of ambiguity, however, since the $c$ 's depend on the choice of the solution in (40). For $d=2$, however, we can make a complete determination by noting that

$$
c_{3}=-\left[1+\phi_{5}\left(4 d^{3}\right)\right]=-\left[1+\phi_{5}(1)\right]
$$

must be even, while the other $c$ 's are odd. This follows from the fact that $\phi_{5}(1)$ is odd since it contains five zero terms, while all the other $\phi_{5}{ }^{\prime} s$ are composed exclusively of an even number of plus and minus ones and must be even. Hence we must have

$$
x+25 w+20 u-10 v \equiv 0 \quad(\bmod 8) .
$$

It is known [4] that $x$ and $w$ are both even or odd according as 2 is a quintic residue of $p$ or not. Hence $x$ and $w$ are both odd and $u$ and $v$ must be of different parity by the second equation in (37). We can let $u$ be even, then by $(37)$

$$
x w \equiv 1-u^{2} \equiv 1+2 u \quad(\bmod 8)
$$


and this implies

$$
w \equiv x+2 u \quad(\bmod 8)
$$

and by (45)

$$
v \equiv x+u \quad(\bmod 4)
$$

or what is the same thing

$$
v \equiv(-1)^{u / 2} x \quad(\bmod 4) .
$$

This determines a unique solution of the system (38) and we can write

$$
2^{(p-1) / 5} \equiv \frac{w\left(125 w^{2}-x^{2}\right)+2(x w+5 u v)(25 w-x+20 u-10 v)}{w\left(125 w^{2}-x^{2}\right)+2(x w+5 u v)(25 w-x-20 u+10 v)} \quad(\bmod p) .
$$

For example let $p=31, x=11, u=2, v=1, w=-1$. We find

$$
2^{(p-1) / 5}=2^{6} \equiv \frac{-1(4)+2(-1)(-6)}{-1(4)+2(-1)(-66)} \equiv \frac{8}{128} \equiv \frac{8}{4} \equiv 2 \quad(\bmod 31),
$$

while

$\left(\begin{array}{c}12 \\ 6\end{array}\right) \equiv \frac{1}{2}[-11-1] \equiv-6 \equiv 25$ and $\left(\begin{array}{c}18 \\ 6\end{array}\right) \equiv \frac{1}{2}[-11+1] \equiv-5 \equiv 26(\bmod 31)$.

Similarly by (38) and (42),

$$
4^{(p-1) / 5} \equiv \frac{w\left(125 w^{2}-x^{2}\right)-2(x w+5 u v)(25 w+x+10 u+20 v)}{w\left(125 w^{2}-x^{2}\right)-2(x w+5 u v)(25 w+x-10 u-20 v)} \quad(\bmod p) .
$$

For $k=8, p=8 n+1=a^{2}+b^{2}$, it is well known that if 2 is a quartic residue of $p$, then $b \equiv 0(\bmod 8)$ and

$$
2^{(p-1) / 8} \equiv(-1)^{b / 8+n} \quad(\bmod p) .
$$

Otherwise since 2 is a quadratic residue, we can use (14) with $D=\sqrt{ } 2$ to obtain

$$
2^{(p-1) / 8} \equiv b / a(\bmod p), \text { where } b / 4 \equiv(-1)^{n} \quad(\bmod 4) .
$$

Expressions for $2^{(p-1) / k}(\bmod p)$ for $k=6,10,12,15,20,24$ and 40 can be easily obtained by combining the above results.

\section{References}

[1] Whiteman, A. L., "Cyclotomy and Jacobsthal Sums", Amer. Jour. of Math. 74 (1952), 89-99.

[2] Jacobsthal, E., "Anwendungen einer Formel aus der.Theorie der quadratischen Reste", Dissertation (Berlin, 1906).

[3] Lehmer, Emma, "On the Number of Solutions of $u^{k}+D \equiv w(\bmod p)$ ", Pacific Jour. of Math. 55 (1955), 103-118.

[4] Lehmer, Emma, "The quintic character of 2 and 3", Duke Math. Journal 18 (1951) 11-18.

Berkeley, Calif., U.S.A. 